\title{
Global Burden of Cataract and Its Association With Socioeconomic Development Status, 1990-2019
}

\section{Xiaotong Han}

Sun Yat-Sen University Zhongshan Ophthalmic Center

Minjie Zou

Sun Yat-Sen University Zhongshan Ophthalmic Center

Zhenzhen Liu

Sun Yat-Sen University Zhongshan Ophthalmic Center

Yi Sun

Third Affiliated Hospital of Sun Yat-Sen University

Charlotte Aimee Young

Nanchang University

Danying Zheng

Sun Yat-Sen University Zhongshan Ophthalmic Center

Guangming Jin ( $\nabla$ jingm@mail2.sysu.edu.cn)

Sun Yat-Sen University https://orcid.org/0000-0001-9994-6338

Research

Keywords: cataract, disease burden, disability-adjusted life year, global eye health.

Posted Date: September 30th, 2021

DOI: https://doi.org/10.21203/rs.3.rs-903175/v1

License: (c) (i) This work is licensed under a Creative Commons Attribution 4.0 International License.

Read Full License 


\section{Abstract}

Background: Cataract is the leading cause of blindness globally and more people will be at risk for this common cause of vision loss in the coming years.

To estimate the disease burden of cataract and evaluate contributions of various risk factors to cataractassociated disability-adjusted life years (DALYs).

Methods: Prevalence of visual impairment due to cataract and disability-adjusted life years of cataract were extracted from the Global Burden of Disease (GBD) study 2019 to explore time trends and annual changes. Regional and country-level socioeconomic indexes were acquired from open databases, and stepwise multiple linear regression was used to evaluate associations between age-standardized rate of DALYs of cataract and potential predictors.

Results: Global Prevalence rate of visual impairment due to cataract rose by $58.45 \%$ from 791.4 per 100,000 population ( $95 \% \mathrm{Cl}$ : 705.2 to 890.0 per 100,000 population) to 1253.9 per 100,000 population $95 \% \mathrm{Cl}$ : 1103.3 to 1417.7 per 100,000 population) in 2019 and DALYs rate of cataract rose by $32.18 \%$ from 65.3 per 100,000 population ( $95 \% \mathrm{Cl}$ : 46.4 to 88.2 per 100,000 population) in 1990 to 86.3 per 100,000 population ( $95 \% \mathrm{Cl}$ : 61.5 to 116.4 per 100,000 population) in 2019 .Stepwise multiple linear regression model showed that higher refractive error prevalence $(\beta=0.036,95 \% \mathrm{Cl}: 0.022,0.050, P<0.001)$, lower number of physicians per 10,000 population ( $\beta=-0.959,95 \% \mathrm{Cl}-1.685,-0.233, P=0.010)$ and lower level of HDI ( $\beta=-134.93,95 \% \mathrm{Cl}$ : $-209.84,-60.02, P=0.001)$ were associated with higher disease burden of cataract.

Conclusions: Substantial increases in the prevalence of visual impairment and DALYs of cataract were observed from 1990 to 2019. Successful global initiatives targeting improving cataract surgical rate and quality, especially in regions with lower socioeconomic status, is a prerequisite to combating this growing burden of cataract in the aging society.

\section{Background}

Cataract is the leading cause of blindness globally and has been a primary focus of many national programs aimed at meeting the Vision 2020 objectives.[1] Despite being treatable with a straightforward and cost-effective surgery, one of the greatest challenges facing ophthalmology globally today remains the unacceptably high backlog of operable cataract blindness, especially in the developing world.[2, 3] It is reported that in 2015 , cataract contributed to $55 \%$ of blindness among adults aged 50 years and older. [4] With the increase in the aging global population, more people will be at risk for this common cause of vision loss in the coming years. $[5,6]$ According to the World Health Organization (WHO) report on vision 2019 , there are at least 1 billion people with preventable moderate or severe distance vision impairment or blindness, including 65.2 million caused by cataract.[5] 
Although many breakthroughs have been made since the inception of Vision 2020 and decreasing blindness prevalence has been achieved during the past decades, the number of blind people continues to increase rapidly. $[4,7]$ The rates of cataract surgery are increasing globally and postoperative outcomes are improving, yet challenges to reducing the cataract burden remain.[3] The Global Burden of Disease (GBD) 2017 Study reported that cataract caused the second largest burden of disability-adjusted life years (DALYs) among eye diseases (8 million), next only to near vision loss.[8] Meanwhile, the DALYs attributed to cataract increased by nearly 30\% from 2007 to 2017.[8] To the best of our knowledge, few studies have specifically assessed the global change in cataract burden thus far. $[9,10]$

By 2030, the number of people worldwide aged 60 years and over is estimated to increase 1.4 billion. Given most people over the age of 60 years will develop cataract, the number of people with this condition will also increase substantially.[11] A successful response to managing this impending challenge requires timely, reliable, quantitative information to design effective interventions aimed at counteracting the disease burden of cataract. Based on the currently available data on the global scale from the GBD, the present study aimed to provide updated information on the global burden of cataract to better inform key stakeholders contributing to the implementation for future health policies.

\section{Methods}

\section{Disease Burden of Cataract}

The disease burden due to cataract (prevalence of visual impairment and DALYs) was obtained from the Global Disease Burden 2019 in the GHDx (http://ghdx.healthdata.org/gbd- results-tool), where the global burden of 369 diseases and injuries of 204 countries and territories were presented and its details of methodology were described.[12] The definition of DALYs is the sum of the years lost due to disability and the years lived with disability by the following formula: DALY number $=$ (Number of death $x$ Standard life expectancy at age of death in years) + (Number of prevalent cases $\times$ Disability weight). [12, 13] The prevalence of visual impairment due to cataract and DALYs of cataract was calculated for populations aged 20 years and above, and DALYs rate was adjusted for population size, with age standardized DALYs rate further adjusted for age structure.

Further analyses were conducted using the data below: (1) the global, GBD regions and GBD super regions numbers, rates and age-standardized rates of DALYs and prevalence of visual impairment due to cataract from 1990 to 2019; (2) country-level age-standardized DALY rate of 2019.

\section{Patient and public involvement}

Patients and the public were not involved in this study.

\section{Country-level indicators}

In order to assess the correlation between disease burden of cataract and potential associated factors, various country level indexes were included for statistical analysis. Former studies gave the conclusion that some of the common ocular diseases, such as glaucoma and refractive error, are positively 
associated with the development of cataract.[14-16] Meanwhile, individuals with diabetes mellitus are at a higher risk of developing cataract. $[17,18]$ The prevalence of diseases mentioned above were extracted from the GBD 2019 Study.[12] Cataract surgical rate, defined as number of cataract surgeries performed annually per million population, indicates the progress in cataract control and was also included for analysis.[19-21]

In terms of included socioeconomic factors, Socio-demographic Index (SDI) is a commonly used index to assess social and economic development level of a country from GHDx, which classify countries into 5 categories as high SDI, middle-high SDI, middle SDI, low-middle SDI and low SDI. Another comparable indicator,Human Development Index (HDI), was extracted from the United Nations Development Programme's (UNDP) database (http://hdr.undp.org/en/data). HDI consists of 4 components across health, education and income dimensions: life expectancy at birth, expected years of schooling, mean years of schooling and gross national income per capita (GNI). The HDI ranged from 0 to 1 , and countries were accordingly divided into 4 groups: very high HDI (HDI 0.800 and above), high HDI (HDI 0.700-0.799), medium HDI (HDI 0.55-0.699) and low HDI (HDI 0.549 and below). Inequality-adjusted HDI was also adapted as it adjusted for the inequal distribution of the HDI within each country. Populations with at least some secondary education (aged 25 and older) is an indicator for evaluation of education level among one country in addition to the 2 components of HDI. Number of physicians, defined by the number of medical doctors per 10,000 population, reflects the healthcare level of a country.

\section{Statistical analysis}

The results are composed of time trends of the prevalence of visual impairment due to cataract and DALYs numbers, DALYs rate, and age standardized DALYs rate attributable to cataract. The global distribution of prevalence of visual impairment due to cataract was downloaded from the GHDx (https://vizhub.healthdata.org/gbd-compare/). One-way analysis of variance (ANOVA) was performed to detect GBD regional as well as GBD super regional difference in age-standardized DALY rate. Scatter plot with regression curve was drawn to evaluate the correlation between age-standardized DALY rates and SDI. Univariate and stepwise linear regression analyses were used to investigate potential correlation with common ocular and endocrine diseases, demographic, socioeconomic and healthcare indicators. Variables significant at a level of $P \leq 0.2$ in univariate regression were included in multiple stepwise regression analysis. Figures were conducted using GraphPad Prism software (V.5.01, GraphPad Software; San Diego, California, USA), while statistical analyses were conducted using Stata MP 15.1 (Stata Corp LP, College Station, Texas, USA). A value of $P<0.05$ was considered statistical significance unless otherwise specified.

\section{Results}

\section{Time trends of disease burden attributable to cataract from 1990 to 2019}


The global trend of prevalence of visual impairment due to cataract and DALYs caused by cataract are presented in Figs. 1 \& 2. Prevalence cases of cataract with visual impairment showed a steady increase by $129.2 \%$, from 4233,6679 (95\%Cl: $3772,8563,4761,5115)$ in 1990 to 9702,2038 (95\%Cl: 8537,0876, $1,0969,6644)$ in 2019. Prevalence rate of visual impairment due to cataract also had an upward trend, rising by $58.45 \%$ from 791.4 per 100,000 population ( $95 \% \mathrm{Cl}$ : 705.2 to 890.0 per 100,000 population) in 1990 to 1253.9 per 100,000 population ( $95 \% \mathrm{Cl}: 1103.3$ to 1417.7 per 100,000 population) in 2019 . Agestandardized prevalence of visual impairment due to cataract fluctuated with its lowest in 1994 (1125.55, 95\%Cl: 1004.97, 1256.61 per 100,000 population) and highest in 2017 (1283.53, 1134.46, 1442.93 per 100,000 population).

In general, trends of DALYs number and DALYs rate were similar to those of the prevalence of visual impairment due to cataract, as DALY numbers increased by $91.2 \%$ from 349,2604 (95\% Cl: 248,1846, $471.9629)$ in 1990 to 667,6281 (95\%Cl: $476,1210,900,6193)$ in 2019 . When it comes to DALYs rate of cataract, it rose by $32.18 \%$ from 65.3 per 100,000 population $(95 \% \mathrm{Cl}: 46.4$ to 88.2 per 100,000 population) in 1990 to 86.3 per 100,000 population ( $95 \% \mathrm{Cl}: 61.5$ to 116.4 per 100,000 population) in 2019. For the age standardized DALY rate, it was uneven throughout the past 30 years with an overall declining trend, ending at 82.94 (95\%Cl: 59.06, 111.75 per 100,000 population) in 2019.

\section{Global distribution of disease burden attributable to cataract in 1990 and 2019 and its annual change}

Figure 3 shows the global distribution of prevalence of visual impairment due to cataract in the years 1990 and 2019. The countries in south Asia and south-east Asia tend to have a higher prevalence of visual impairment due to cataract in 1990 and 2019. Indonesia had the most severe disease burden in the year of 1990 (1972.50, 95\% Cl: 1799.04, 2158.74 per 100,000 population), while Thailand surpassed Indonesia in 2019 (3231.99, 95\% Cl: 2960.79, 3497.79 per 100,000 population). The annual change for prevalence of visual impairment due to cataract was positive in most countries, except for some countries located in the Middle East and Africa. As for the comparison of age-standardized prevalence of visual impairment due to cataract and DALYs rate between 1990 and 2019 among GBD super regions (Table 1), the global prevalence of visual impairment due to cataract in 2019 raised by $4.98 \%(95 \% \mathrm{Cl}$ : $4.86 \%, 5.11 \%$ ) compared to that of 1990 . Disease burden of most geographical regions tended to decline, with the exception of the east Asia region, whose DALYs rate increased by $14.13 \%$ (95\% Cl: $13.89 \%$, $14.36 \%)$ to $967.90(95 \% \mathrm{Cl}: 840.60,1104.44)$ per 100,000 population in the year of 2019 . The age standardized DALYs rates of 2019 among the selected GBD super regions, on the other hand, were all lower than those of 1990. 
Table 1

Age-standardized prevalence of visual impairment due to cataract in 1990 and 2019 and its time trends from 1990 to 2019.

\begin{tabular}{|c|c|c|c|c|c|c|}
\hline \multirow[t]{2}{*}{ Location } & \multicolumn{3}{|l|}{ Prevalence (95\% UI) } & \multicolumn{3}{|c|}{ DALYS (95\%UI) } \\
\hline & 1990 & 2019 & $\begin{array}{l}\text { Percentage } \\
\text { change } \\
\text { 1990-2019 }\end{array}$ & 1990 & 2019 & $\begin{array}{l}\text { Percentage } \\
\text { change } \\
1990-2019\end{array}$ \\
\hline Global & $\begin{array}{l}1150.56(1027.31 \\
1287.40)\end{array}$ & $\begin{array}{l}1207.88 \\
(1065.04, \\
1361.26)\end{array}$ & $\begin{array}{l}4.98(4.86 \\
5.11)\end{array}$ & $\begin{array}{l}93.17 \\
(66.14 \\
125.32)\end{array}$ & $\begin{array}{l}82.94 \\
(59.06 \\
111.75)\end{array}$ & $\begin{array}{l}-10.98 \\
(-11.63 \\
-10.35)\end{array}$ \\
\hline Low SDI & $\begin{array}{l}2016.86(1810.94 \\
2241.28)\end{array}$ & $\begin{array}{l}1966.87 \\
(1753.94 \\
2205.73)\end{array}$ & $\begin{array}{l}-2.48(-2.55 \\
-2.41)\end{array}$ & $\begin{array}{l}180.68 \\
(128.63 \\
244.99)\end{array}$ & $\begin{array}{l}154.48 \\
(108.77 \\
206.59)\end{array}$ & $\begin{array}{l}-14.50 \\
(-15.02 \\
-13.99)\end{array}$ \\
\hline $\begin{array}{l}\text { Low-middle } \\
\text { SDI }\end{array}$ & $\begin{array}{l}2495.12(2234.99 \\
2780.44)\end{array}$ & $\begin{array}{l}2182.06 \\
(1937.04, \\
2455.86)\end{array}$ & $\begin{array}{l}-12.55 \\
(-12.68 \\
-12.42)\end{array}$ & $\begin{array}{l}216.76 \\
(153.53 \\
292.32)\end{array}$ & $\begin{array}{l}160.43 \\
(114.24 \\
215.23)\end{array}$ & $\begin{array}{l}-25.99 \\
(-26.58 \\
-25.40)\end{array}$ \\
\hline Middle SDI & $\begin{array}{l}1634.59 \text { (1464.80, } \\
1816.28)\end{array}$ & $\begin{array}{l}1541.64 \\
(1373.65, \\
1723.02)\end{array}$ & $\begin{array}{l}-5.69(-5.80 \\
-5.57)\end{array}$ & $\begin{array}{l}139.91 \\
(99.06 \\
188.58)\end{array}$ & $\begin{array}{l}107.61 \\
(75.96 \\
144.28)\end{array}$ & $\begin{array}{l}-23.09 \\
(-23.79 \\
-22.39)\end{array}$ \\
\hline $\begin{array}{l}\text { High- } \\
\text { middle SDI }\end{array}$ & $\begin{array}{l}775.10(678.01 \\
876.07)\end{array}$ & $\begin{array}{l}893.60 \\
(774.42 \\
1013.51)\end{array}$ & $\begin{array}{l}15.29 \\
(15.04, \\
15.54)\end{array}$ & $\begin{array}{l}54.05 \\
(38.25, \\
71.81)\end{array}$ & $\begin{array}{l}53.72 \\
(38.45 \\
72.57)\end{array}$ & $\begin{array}{l}-0.61(-0.86 \\
-0.42)\end{array}$ \\
\hline High SDI & $\begin{array}{l}354.32(305.76 \\
407.33)\end{array}$ & $\begin{array}{l}357.01 \\
(306.85 \\
409.46)\end{array}$ & $\begin{array}{l}0.78(0.69 \\
0.88)\end{array}$ & $\begin{array}{l}21.86 \\
(15.50 \\
29.71)\end{array}$ & $\begin{array}{l}20.57 \\
(14.49 \\
28.10)\end{array}$ & $\begin{array}{l}-5.90(-6.97 \\
-4.95)\end{array}$ \\
\hline $\begin{array}{l}\text { Central } \\
\text { Europe }\end{array}$ & $\begin{array}{l}399.38(335.02 \\
468.77)\end{array}$ & $\begin{array}{l}391.55 \\
(326.74 \\
462.98)\end{array}$ & $\begin{array}{l}-1.96(-2.10 \\
-1.83)\end{array}$ & $\begin{array}{l}21.26 \\
(14.81 \\
28.96)\end{array}$ & $\begin{array}{l}19.36 \\
(13.34 \\
26.63)\end{array}$ & $\begin{array}{l}-8.94 \\
(-10.23 \\
-7.76)\end{array}$ \\
\hline Australasia & $\begin{array}{l}356.57(307.67 \\
406.66)\end{array}$ & $\begin{array}{l}355.89 \\
(301.23 \\
410.70)\end{array}$ & $\begin{array}{l}-0.19(-0.15 \\
-0.02)\end{array}$ & $\begin{array}{l}21.32 \\
(14.83 \\
29.03)\end{array}$ & $\begin{array}{l}20.30 \\
(14.25, \\
27.82)\end{array}$ & $\begin{array}{l}-4.78(-5.78 \\
-3.92)\end{array}$ \\
\hline $\begin{array}{l}\text { Central } \\
\text { Asia }\end{array}$ & $\begin{array}{l}1219.74(1043.81 \\
1397.11)\end{array}$ & $\begin{array}{l}1152.73 \\
(977.42, \\
1334.76)\end{array}$ & $\begin{array}{l}-5.49(-5.62 \\
-5.37)\end{array}$ & $\begin{array}{l}77.49 \\
(54.76 \\
104.16)\end{array}$ & $\begin{array}{l}66.22 \\
(45.77 \\
89.99)\end{array}$ & $\begin{array}{l}-14.54 \\
(-15.35 \\
-13.77)\end{array}$ \\
\hline $\begin{array}{l}\text { Central } \\
\text { Latin } \\
\text { America }\end{array}$ & $\begin{array}{l}1101.92(966.45 \\
1248.99)\end{array}$ & $\begin{array}{l}942.18 \\
(816.67 \\
1077.83)\end{array}$ & $\begin{array}{l}-14.50 \\
(-14.71 \\
-14.29)\end{array}$ & $\begin{array}{l}90.64 \\
(64.33 \\
121.65)\end{array}$ & $\begin{array}{l}64.70 \\
(46.00 \\
87.16)\end{array}$ & $\begin{array}{l}-28.62 \\
(-29.56 \\
-27.69)\end{array}$ \\
\hline $\begin{array}{l}\text { Tropical } \\
\text { Latin } \\
\text { America }\end{array}$ & $\begin{array}{l}1143.56(1014.81 \\
1280.30)\end{array}$ & $\begin{array}{l}975.80 \\
(857.20 \\
1097.80)\end{array}$ & $\begin{array}{l}-14.58 \\
(-14.79 \\
-14.38)\end{array}$ & $\begin{array}{l}96.82 \\
(68.48 \\
130.47)\end{array}$ & $\begin{array}{l}70.86 \\
(50.45 \\
94.07)\end{array}$ & $\begin{array}{l}-26.81 \\
(-27.71 \\
-25.93)\end{array}$ \\
\hline Caribbean & $\begin{array}{l}\text { 690.17 (591.39, } \\
796.14)\end{array}$ & $\begin{array}{l}620.33 \\
(525.97 \\
721.44)\end{array}$ & $\begin{array}{l}-10.12 \\
(-10.35 \\
-9.90)\end{array}$ & $\begin{array}{l}56.30 \\
(39.63 \\
76.84)\end{array}$ & $\begin{array}{l}42.90 \\
(30.02 \\
58.37)\end{array}$ & $\begin{array}{l}-23.80 \\
(-24.94 \\
-22.69)\end{array}$ \\
\hline
\end{tabular}




\begin{tabular}{|c|c|c|c|c|c|c|}
\hline Location & Prevalence ( $95 \%$ UI) & & & DALYS ( & \%UI) & \\
\hline $\begin{array}{l}\text { Southern } \\
\text { sub- } \\
\text { Saharan } \\
\text { Africa }\end{array}$ & $\begin{array}{l}1082.82(965.44 \\
1207.09)\end{array}$ & $\begin{array}{l}860.85 \\
(763.12 . \\
967.27)\end{array}$ & $\begin{array}{l}-20.50 \\
(-20.74 \\
-20.26)\end{array}$ & $\begin{array}{l}119.28 \\
(84.16, \\
164.15)\end{array}$ & $\begin{array}{l}80.48 \\
(57.65 \\
110.15)\end{array}$ & $\begin{array}{l}-32.53 \\
(-33.38 \\
-31.69)\end{array}$ \\
\hline $\begin{array}{l}\text { Eastern } \\
\text { Europe }\end{array}$ & $\begin{array}{l}531.04(451.57 \\
618.71)\end{array}$ & $\begin{array}{l}506.85 \\
(428.34 \\
588.70)\end{array}$ & $\begin{array}{l}-4.56(-4.74 \\
-4.38)\end{array}$ & $\begin{array}{l}33.49 \\
(23.53 \\
44.81)\end{array}$ & $\begin{array}{l}28.94 \\
(20.29, \\
39.35)\end{array}$ & $\begin{array}{l}-13.59 \\
(-14.79 \\
-12.44)\end{array}$ \\
\hline $\begin{array}{l}\text { Southern } \\
\text { Latin } \\
\text { America }\end{array}$ & $\begin{array}{l}564.67(484.69, \\
649.62)\end{array}$ & $\begin{array}{l}544.05 \\
(462.34 \\
627.92)\end{array}$ & $\begin{array}{l}-3.65(-3.81 \\
-3.50)\end{array}$ & $\begin{array}{l}38.56 \\
(26.81 \\
51.96)\end{array}$ & $\begin{array}{l}32.50 \\
(22.74, \\
44.10)\end{array}$ & $\begin{array}{l}-15.72 \\
(-16.90 \\
-14.58)\end{array}$ \\
\hline $\begin{array}{l}\text { Andean } \\
\text { Latin } \\
\text { America }\end{array}$ & $\begin{array}{l}1764.55 \\
(1551.78,1988.38)\end{array}$ & $\begin{array}{l}1447.36 \\
(1266.22 \\
1648.07)\end{array}$ & $\begin{array}{l}-17.96 \\
(-18.16 \\
-17.80)\end{array}$ & $\begin{array}{l}140.25 \\
(99.00 \\
188.37)\end{array}$ & $\begin{array}{l}96.22 \\
(68.28 \\
129.43)\end{array}$ & $\begin{array}{l}-31.39 \\
(-32.17 \\
-30.63)\end{array}$ \\
\hline $\begin{array}{l}\text { Southeast } \\
\text { Asia }\end{array}$ & $\begin{array}{l}3110.31(2827.96 \\
3422.57)\end{array}$ & $\begin{array}{l}2613.61 \\
(2391.13 \\
2860.70)\end{array}$ & $\begin{array}{l}-15.97 \\
(-16.10 \\
-15.84)\end{array}$ & $\begin{array}{l}279.06 \\
(198.51 \\
378.15)\end{array}$ & $\begin{array}{l}195.67 \\
(138.40 \\
262.38)\end{array}$ & $\begin{array}{l}-29.88 \\
(-30.42 \\
-29.35)\end{array}$ \\
\hline $\begin{array}{l}\text { Western } \\
\text { Europe }\end{array}$ & $\begin{array}{l}448.19 \text { (383.96, } \\
519.03)\end{array}$ & $\begin{array}{l}440.10 \\
(376.20 \\
507.66)\end{array}$ & $\begin{array}{l}-1.81(-1.93, \\
-1.68)\end{array}$ & $\begin{array}{l}26.68 \\
(18.64 \\
36.59)\end{array}$ & $\begin{array}{l}25.31 \\
(17.74 \\
34.81)\end{array}$ & $\begin{array}{l}-5.13(-6.04 \\
-4.33)\end{array}$ \\
\hline $\begin{array}{l}\text { High- } \\
\text { income } \\
\text { Asia Pacific }\end{array}$ & $\begin{array}{l}313.83(271.38 \\
358.06)\end{array}$ & $\begin{array}{l}310.34 \\
(267.86, \\
356.64)\end{array}$ & $\begin{array}{l}-1.11(-1.23, \\
-1.00)\end{array}$ & $\begin{array}{l}21.09 \\
(14.83 \\
28.65)\end{array}$ & $\begin{array}{l}19.50 \\
(13.59 \\
26.89)\end{array}$ & $\begin{array}{l}-7.54(-8.75, \\
-6.45)\end{array}$ \\
\hline South Asia & $\begin{array}{l}3098.49(2773.45 \\
3456.87)\end{array}$ & $\begin{array}{l}2663.74 \\
(2359.84 \\
3005.09)\end{array}$ & $\begin{array}{l}-14.03 \\
(-14.15 \\
-13.91)\end{array}$ & $\begin{array}{l}273.27 \\
(194.60 \\
366.72)\end{array}$ & $\begin{array}{l}198.39 \\
(142.54 \\
264.15)\end{array}$ & $\begin{array}{l}-27.40 \\
(-27.93 \\
-26.87)\end{array}$ \\
\hline $\begin{array}{l}\text { High- } \\
\text { income } \\
\text { North } \\
\text { America }\end{array}$ & $\begin{array}{l}289.16(249.35 \\
330.20)\end{array}$ & $\begin{array}{l}288.60 \\
(249.38 \\
330.01)\end{array}$ & $\begin{array}{l}-0.19(-0.25 \\
-0.15)\end{array}$ & $\begin{array}{l}18.06 \\
(12.84, \\
24.71)\end{array}$ & $\begin{array}{l}17.42 \\
(12.34, \\
23.78)\end{array}$ & $\begin{array}{l}-3.54(-4.50, \\
-2.74)\end{array}$ \\
\hline East Asia & $\begin{array}{l}848.08(744.35, \\
954.58)\end{array}$ & $\begin{array}{l}967.90 \\
(840.60 \\
1104.44)\end{array}$ & $\begin{array}{l}14.13 \\
(13.89, \\
14.36)\end{array}$ & $\begin{array}{l}63.90 \\
(45.00 \\
86.66)\end{array}$ & $\begin{array}{l}57.37 \\
(40.44 \\
77.80)\end{array}$ & $\begin{array}{l}-10.22 \\
(-10.99 \\
-9.49)\end{array}$ \\
\hline $\begin{array}{l}\text { North } \\
\text { Africa and } \\
\text { Middle East }\end{array}$ & $\begin{array}{l}1775.75(1566.58 \\
2007.55)\end{array}$ & $\begin{array}{l}1534.09 \\
(1332.42, \\
1756.61)\end{array}$ & $\begin{array}{l}-13.61 \\
(-13.77 \\
-13.45)\end{array}$ & $\begin{array}{l}143.15 \\
(101.05, \\
195.59)\end{array}$ & $\begin{array}{l}98.21 \\
(69.44, \\
134.06)\end{array}$ & $\begin{array}{l}-31.39 \\
(-32.16 \\
-30.63)\end{array}$ \\
\hline Oceania & $\begin{array}{l}2467.72(2179.42 \\
2784.63)\end{array}$ & $\begin{array}{l}2463.23 \\
(2174.16 \\
2763.30)\end{array}$ & $\begin{array}{l}-0.18(-0.20 \\
-0.17)\end{array}$ & $\begin{array}{l}181.65 \\
(128.82, \\
242.25)\end{array}$ & $\begin{array}{l}163.72 \\
(116.76, \\
222.47)\end{array}$ & $\begin{array}{l}-9.87 \\
(-10.31 \\
-9.44)\end{array}$ \\
\hline $\begin{array}{l}\text { Central sub- } \\
\text { Saharan } \\
\text { Africa }\end{array}$ & $\begin{array}{l}425.58(365.03 \\
493.53)\end{array}$ & $\begin{array}{l}413.80 \\
(345.05 \\
487.15)\end{array}$ & $\begin{array}{l}-2.77(-2.93 \\
-2.61)\end{array}$ & $\begin{array}{l}32.86 \\
(22.99 \\
45.35)\end{array}$ & $\begin{array}{l}26.13 \\
(18.05, \\
35.82)\end{array}$ & $\begin{array}{l}-20.48 \\
(-21.90 \\
-19.11)\end{array}$ \\
\hline
\end{tabular}

SDI, sociodemographic index; UI uncertainty interval. 


\begin{tabular}{|c|c|c|c|c|c|c|}
\hline Location & Prevalence $(95 \% \mathrm{UI})$ & & & DALYS ( & $\% \mathrm{UI})$ & \\
\hline $\begin{array}{l}\text { Eastern } \\
\text { sub- } \\
\text { Saharan } \\
\text { Africa }\end{array}$ & $\begin{array}{l}1651.44(1490.61 \\
1822.16)\end{array}$ & $\begin{array}{l}1489.44 \\
(1337.15, \\
1658.66)\end{array}$ & $\begin{array}{l}-9.81(-9.95 \\
-9.67)\end{array}$ & $\begin{array}{l}156.81 \\
(112.06, \\
215.18)\end{array}$ & $\begin{array}{l}129.15 \\
(91.78, \\
176.19)\end{array}$ & $\begin{array}{l}-17,64 \\
(-18.24 \\
-17.05)\end{array}$ \\
\hline $\begin{array}{l}\text { Western } \\
\text { sub- } \\
\text { Saharan } \\
\text { Africa }\end{array}$ & $\begin{array}{l}1996.56(1798.53 \\
2215.80)\end{array}$ & $\begin{array}{l}2155.19 \\
(1912.45, \\
2412.08)\end{array}$ & $\begin{array}{l}7.95(7.83 \\
8.06)\end{array}$ & $\begin{array}{l}165.62 \\
(117.94 \\
224.66)\end{array}$ & $\begin{array}{l}152.14 \\
(107.71 \\
205.16)\end{array}$ & $\begin{array}{l}-8.14(-9.57, \\
-7.73)\end{array}$ \\
\hline
\end{tabular}

\section{Global cataract disease burden by SDI level}

The changes in age-standardized DALY rates among GBD super regions by SDI from 1990 to 2019 are given in Fig. 4. Disease burden attributable to cataract of all regions tend to decrease throughout the past 30 years, with region of higher SDI having less disease burden due to cataract. Individuals dwelling in southeast Asia and south Asia were at a higher risk of vision loss caused by cataract, whereas people in high-income North America, high-income Asian Pacific, Western Europe and Australasia were less likely to develop vision loss due to cataract.

\section{Country-level analysis of disease burden attributable to cataract with various factors}

As shown in Table 2, glaucoma and refractive error were positively associated with the disease burden of cataract, accounting for $16.95 \%$ and $14.30 \%$ of the global variation, respectively. $(P<0.001)$ Diabetes mellitus, on the other hand, did not show any association with disease burden of cataract. 
Table 2

Potential associated factors for national disease burden due to cataract

\begin{tabular}{|c|c|c|c|c|c|c|c|}
\hline \multirow[t]{2}{*}{ Factors } & \multicolumn{3}{|c|}{ Univariate linear regression } & \multicolumn{2}{|c|}{$\begin{array}{l}\text { Multiple linear } \\
\text { regression } 1\end{array}$} & \multicolumn{2}{|c|}{$\begin{array}{l}\text { Multiple linear } \\
\text { regression } 2\end{array}$} \\
\hline & $\beta(95 \% \mathrm{Cl})$ & $\mathrm{R}^{2}$ & $\mathbf{P}$ & $\beta(95 \% \mathrm{Cl})$ & $\mathbf{P}$ & $\beta(95 \% \mathrm{Cl})$ & $\mathbf{P}$ \\
\hline $\begin{array}{l}\text { Glaucoma } \\
\text { prevalence }\end{array}$ & $\begin{array}{l}0.279 \\
(0.193 \\
0.364)\end{array}$ & 0.1695 & $<.001$ & - & - & - & - \\
\hline $\begin{array}{l}\text { Refractive error } \\
\text { prevalence }\end{array}$ & $\begin{array}{l}0.038 \\
(0.025 \\
0.051)\end{array}$ & 0.1430 & $<0.001$ & $\begin{array}{l}0.036 \\
(0.022, \\
0.050)\end{array}$ & $<0.001$ & $\begin{array}{l}0.032 \\
(0.019, \\
0.046)\end{array}$ & $\begin{array}{l}<.001 \\
0.00\end{array}$ \\
\hline $\begin{array}{l}\text { Diabetes } \\
\text { mellitus } \\
\text { prevalence }\end{array}$ & $\begin{array}{l}0.002 \\
(-0.0006 \\
0.004)\end{array}$ & 0.0102 & 0.151 & - & - & - & - \\
\hline SDI & $\begin{array}{l}-159.55 \\
(-197.16 \\
-121.95)\end{array}$ & 0.2573 & $\begin{array}{l}< \\
0.001\end{array}$ & - & - & - & - \\
\hline CSR & $\begin{array}{l}-0.006 \\
(-0.010 \\
-0.002)\end{array}$ & 0.0550 & 0.002 & - & - & - & - \\
\hline $\begin{array}{l}\text { Population with } \\
\text { at least some } \\
\text { secondary } \\
\text { education }\end{array}$ & $\begin{array}{l}-1.101 \\
(-1.380 \\
-0.822)\end{array}$ & 0.2817 & $\begin{array}{l}< \\
0.001\end{array}$ & - & - & - & - \\
\hline $\begin{array}{l}\text { Number of } \\
\text { Physicians }\end{array}$ & $\begin{array}{l}-1.678 \\
(-2.156 \\
-1.199)\end{array}$ & 0.1298 & $\begin{array}{l}< \\
0.001\end{array}$ & $\begin{array}{l}-0.959 \\
(-1.685 \\
-0.233)\end{array}$ & 0.010 & $\begin{array}{l}-0.982 \\
(-1.587 \\
-0.196)\end{array}$ & 0.012 \\
\hline HDI & $\begin{array}{l}-86.161 \\
(-123.207 \\
-49.115)\end{array}$ & 0.1103 & $\begin{array}{l}<.001 \\
0.001\end{array}$ & $\begin{array}{l}-134.93 \\
(-209.84 \\
-60.02)\end{array}$ & 0.001 & - & - \\
\hline$|A-H D|$ & $\begin{array}{l}-80.531 \\
(-106.725 \\
-54.338)\end{array}$ & 0.1781 & $<.001$ & - & - & - & - \\
\hline $\begin{array}{l}\text { Life Expectancy } \\
\text { at birth }\end{array}$ & $\begin{array}{l}-0.577 \\
(-1.073 \\
-0.081)\end{array}$ & 0.0301 & 0.023 & - & - & - & - \\
\hline
\end{tabular}

SDI, sociodemographic index; CSR, cataract surgery rate; HDI, human development index; IA-HDI, inequality-adjusted human development index; GNI per capita, gross national income per capita.

Multiple linear regression 1: adjusted for glaucoma prevalence, refractive error prevalence, diabetes mellitus prevalence, CSR, population with at least some secondary education, number of physicians and HDI. Multiple linear regression 2: adjusted for glaucoma prevalence, refractive error prevalence, diabetes mellitus prevalence, CSR, population with at least some secondary education, number of physicians, life expectancy at birth, expected years of schooling, mean years of schooling and GNI per capita. 


\begin{tabular}{|c|c|c|c|c|c|c|c|}
\hline \multirow[t]{2}{*}{ Factors } & \multicolumn{3}{|c|}{ Univariate linear regression } & \multicolumn{2}{|c|}{$\begin{array}{l}\text { Multiple linear } \\
\text { regression } 1\end{array}$} & \multicolumn{2}{|c|}{$\begin{array}{l}\text { Multiple linear } \\
\text { regression } 2\end{array}$} \\
\hline & $\beta(95 \% \mathrm{Cl})$ & $\mathrm{R}^{2}$ & $\mathbf{P}$ & $\beta(95 \% \mathrm{Cl})$ & $\mathbf{P}$ & $\beta(95 \% \mathrm{Cl})$ & $\mathbf{P}$ \\
\hline $\begin{array}{l}\text { Expected years } \\
\text { of schooling }\end{array}$ & $\begin{array}{l}-4.723 \\
(-6.649 \\
-2.797)\end{array}$ & 0.1212 & $<.001$ & - & - & $\begin{array}{l}-5.183 \\
(-9.564 \\
-0.802)\end{array}$ & 0.021 \\
\hline $\begin{array}{l}\text { Mean years of } \\
\text { schooling }\end{array}$ & $\begin{array}{l}-6.899 \\
(-9.037 \\
-4.761)\end{array}$ & 0.1927 & $<.001$ & - & - & $\begin{array}{l}-2.782 \\
(-7.141 \\
1.577)\end{array}$ & 0.209 \\
\hline GNI per capita & $\begin{array}{l}-0.001 \\
(-0.002 \\
-0.0005)\end{array}$ & 0.1132 & $\dot{0} 001$ & - & - & - & - \\
\hline \multicolumn{8}{|c|}{$\begin{array}{l}\text { SDI, sociodemographic index; CSR, cataract surgery rate; HDI, human development index; IA-HDI, } \\
\text { inequality-adjusted human development index; GNI per capita, gross national income per capita. }\end{array}$} \\
\hline \multicolumn{8}{|c|}{$\begin{array}{l}\text { Multiple linear regression 1: adjusted for glaucoma prevalence, refractive error prevalence, diabetes } \\
\text { mellitus prevalence, CSR, population with at least some secondary education, number of physicians } \\
\text { and HDI. Multiple linear regression 2: adjusted for glaucoma prevalence, refractive error prevalence, } \\
\text { diabetes mellitus prevalence, CSR, population with at least some secondary education, number of } \\
\text { physicians, life expectancy at birth, expected years of schooling, mean years of schooling and GNI per } \\
\text { capita. }\end{array}$} \\
\hline
\end{tabular}

As for socioeconomic indicators, scatter plot with regression curve indicated that the age-standardized DALY rate of cataract was negatively correlated to SDI level (Fig. 5). In the stepwise multiple linear regression model, higher refractive error prevalence ( $\beta=0.036,95 \% \mathrm{Cl}: 0.022,0.050, P<0.001)$, a smaller number of physicians $(\beta=-0.959,95 \% \mathrm{Cl}:-1.685,-0.233, P=0.010)$ and lower level of $\mathrm{HDI}(\beta=-134.93,95 \%$ Cl: $-209.84,-60.02, P=0.001)$ were associated with higher disease burden of cataract. Meanwhile, higher refractive error prevalence $(\beta=0.032,95 \% \mathrm{Cl}: 0.019,0.046, P<0.001)$ and a smaller number of physicians ( $\beta=-0.982,95 \% \mathrm{Cl}:-1.587,-0.196, P=0.012)$ were also correlated with more severe disease burden in multiple regression model 2 , together with shorter expected years of schooling ( $\beta=-5.183,95 \% \mathrm{Cl}$ : -9.564 , $-0.802, P=0.021)$.

\section{Discussion}

Our results shows that the global prevalence of visual impairment due to cataract and DALYs of cataract showed an increasing trend from 1990 to 2019, with higher burden was found in low socio-economic areas. Severer burden of visual impairment due to cataract is associated with lower SDI, lower HDI, smaller number of physicians and higher refractive error prevalence. Our findings are consistent with previous GBD studies, $[9,10]$ and further emphasize the importance of implementing strategies to increase coverage and reduce gaps to eliminate the rising cataract burden globally.

The crude prevalence of visual impairment due to cataract and DALYs of cataract increased steadily from 1990 to 2017, but showed a decreasing trend afterwards until 2019; this decreasing trend could more 
clearly be seen in age-standardized rates (Fig. 1\&2). Meanwhile, despite the increase of age-standardized prevalence of visual impairment due to cataract by $4.98 \%$ during the past 30 years, the age-standardized DALY of cataract consistently decreased (decreased by 10.98\%). Taken together, this suggests a global health improvement in cataract, especially after 2017. However, the overall global burden of cataract remains high, which can be partially explained by taking into account the aging and growth of the population.

Despite the overall improvement in global cataract care, the socioeconomic disparity in cataract burden is a major challenge for reducing global cataract blindness. Previous studies have reported higher cataract prevalence and lower cataract surgical rates in developing and un-developed countries.[22] The GBD Studies have also reported consistently increasing socioeconomic-associated inequality in global cataract burden in the last decades.[10] In our study, the age-standardized prevalence of visual impairment due to cataract was highest in low-middle SDI regions and lowest in high SDI regions in both 1990 and 2019. The greatest declines in age-standardized DALYs rate of cataract between 1990 and 2019 were in Southern sub-Saharan Africa, Andean Latin America and Southeast Asia, while the greatest increase was in East Asia and Western sub-Saharan Africa. With more than $20 \%$ of the total world population, the East Asia regions contribute to largest increase in cataract burden and are in urgent need for future effective strategies to combat the upcoming increasing challenge of cataract burden. Additionally, the age-standardized DALYs of cataract in 2019 were significantly reduced in all regions as compared to 1990, with the greatest decline seen in Low-middle SDI regions. The percentage change in age-standardized DALY was largest in Southern sub-Saharan Africa, North Africa and Middle East, and Andean Latin America (all over 30\%). In comparison, the decreases in East Asia and Western sub-Saharan Africa were only modest (10.22\% and $8.14 \%)$, and below the average global decreasing level.

Consistent with previous studies, our study showed that lower number of physicians and less education were significant risk factors for higher cataract burden.[23-25] It is noteworthy that higher refractive error prevalence was also found to be positively related to a higher national disease burden due to cataract, even after adjusting for all other covariates. One possible reason may lies in that with the increasing prevalence of refractive error,the complex cataract cases due to myopia increase.[26] Various studies have found that individuals with high myopia are more likely to develop cataract.[26, 27] The Blue Mountains Eye Study found that even participants with low myopia (-1 to $-3.5 \mathrm{D})$ had higher risk of posterior subcapsular cataract.[28] A meta-analysis reported that myopia was associated with a higher risk of nuclear and posterior subcortical cataract.[29] However, advances in surgical techniques for cataract, coupled with improvements in intraocular lens design and the increased availability of low-cost, high-quality intraocular lens, have led to significant improvements in patient accessibility and surgical outcomes. In the last decades, the increase in the prevalence of visual impairment due to cataract and its associated disease burden warrants further investigation and monitoring of cataract on a national and global basis, as well as the identification of determinants for further increase. Implementation of currently known and effective treatments of cataract are expected to contribute significant productivity gains to the global economy at a fraction of the estimated costs to deliver them.[30] Increasing the quantity and quality of cataract surgery services, and tailored blindness-prevention programs to meet the needs of 
local population and resources remains the most important solutions to the existing and impending cataract burden.

The present study provides stakeholders with estimates at regional and global level over 20 years to help monitor the effectiveness of interventions regarding cataract through time. However, some limitations should be noted. The study was subject to the limitations that the GBD 2019 study had noted in their reports, including statistical assumption and data sources.[12, 31] Specifically for cataract, the use of aggregated data at a country level would be a source of bias, due to geographic variations in DALY estimates. In addition, potential associated factors for cataract burden including cost, ophthalmologist numbers, facilities and surgical quality were not available, $[25,32]$ and future studies investigating these important determinants for cataract burden can provide a more comprehensive assessment and support for strategic planning.

\section{Conclusion}

In summary, cataract is still an important contributor to global disease burden which is likely to continue to pose a great challenge in health care with population aging and growth. Successful global initiatives targeting improving cataract surgical rate and quality, especially in regions with lower socioeconomic status, is a prerequisite to combating this growing burden of cataract in the aging society.

\section{Declarations}

\section{Ethics Approval and Consent to participate:}

Not applicable.

\section{Consent for publication}

Not applicable.

\section{Availability of Data and Materials}

Data are available on request. Any request for data can be made to the corresponding author and are subject to ethics approval.

\section{Conflict of Interest:}

The authors have no financial or other conflicts of interest concerning this study.

\section{Funding:}

This work was supported by National Natural Science Foundation of China $(81873673,81900841)$. X.H. receives support from the China Postdoctoral Science Foundation (2019TQ0365). 


\section{Authors' contributions:}

Conception and design: DZ, GJ;

Analysis and interpretation: $\mathrm{XH}, \mathrm{MZ}, \mathrm{ZL}, \mathrm{YS}, \mathrm{AC}$;

Writing of the article: $\mathrm{XH}, \mathrm{MZ}, \mathrm{ZL}$;

Critical revision of the article: GJ, DZ;

Data collection: $\mathrm{XH}, \mathrm{MZ}, \mathrm{ZL}$ YS;

Administrative, technical or logistical support: GJ, DZ.

\section{Acknowledgement:}

Not applicable.

\section{References}

1. Bourne RRA. Vision 2020: where are we? Curr Opin Ophthalmol. 2020;31:81-4.

2. Hashemi H, Pakzad R, Yekta A, Aghamirsalim M, Pakbin M, Ramin S, Khabazkhoob M. Global and regional prevalence of age-related cataract: a comprehensive systematic review and meta-analysis. Eye. 2020;34:1357-70.

3. Lee CM, Afshari NA. The global state of cataract blindness. Curr Opin Ophthalmol. 2017;28:98-103.

4. Flaxman SR, Bourne RRA, Resnikoff S, Ackland P, Braithwaite T, Cicinelli MV, Das A, Jonas JB, Keeffe J, Kempen JH, et al. Global causes of blindness and distance vision impairment 1990-2020: a systematic review and meta-analysis. The Lancet Global health. 2017;5:e1221-34.

5. Organization WH: World Report on Vision. 2019, Magnitude, temporal trends, and projections of the global prevalence of blindness and distance and near vision impairment: a systematic review and meta-analysis.

6. Bourne RRA, Flaxman SR, Braithwaite T, Cicinelli MV, Das A, Jonas JB, Keeffe J, Kempen JH, Leasher $\mathrm{J}$, Limburg $\mathrm{H}$, et al. Magnitude, temporal trends, and projections of the global prevalence of blindness and distance and near vision impairment: a systematic review and meta-analysis. The Lancet Global health. 2017;5:e888-97.

7. Taylor HR. Global Blindness: The Progress We Are Making and Still Need to Make. Asia-Pacific journal of ophthalmology. 2019;8:424-8.

8. DALYs GBD, Collaborators H. Global, regional, and national disability-adjusted life-years (DALYs) for 359 diseases and injuries and healthy life expectancy (HALE) for 195 countries and territories, 19902017: a systematic analysis for the Global Burden of Disease Study 2017. Lancet. 2018;392:1859922. 
9. Lou L, Ye X, Xu P, Wang J, Xu Y, Jin K, Ye J. Association of Sex With the Global Burden of Cataract. JAMA ophthalmology. 2018;136:116-21.

10. Lou L, Wang J, Xu P, Ye X, Ye J. Socioeconomic Disparity in Global Burden of Cataract: An Analysis for 2013 With Time Trends Since 1990. Am J Ophthalmol. 2017;180:91-6.

11. Organization WH: Decade of Healthy Ageing. 2019, https://www.who.int/docs/defaultsource/documents/decade-of-health-ageing/decade-ageing-proposal-en.pdf? Status=Temp\&sfvrsn=b0a7b5b1_12.

12. Global burden of 369 diseases and injuries in 204 countries and territories, 1990-2019: a systematic analysis for the Global Burden of Disease Study 2019. Lancet (London, England) 2020, 396:12041222.

13. Shuaib AM, El Sayed Y, Kamal A, El Sanabary Z, Elhilali H. Transscleral sutureless intraocular lens versus retropupillary iris-claw lens fixation for paediatric aphakia without capsular support: a randomized study. Acta ophthalmologica. 2019;97:e850-9.

14. Zhang Y, Jin G, Fan M, Lin Y, Wen X, Li Z, Zeng P, Zheng D, Lan Y. Time trends and heterogeneity in the disease burden of glaucoma, 1990-2017: a global analysis. Journal of global health. 2019;9:020436.

15. Shah M, Law G, Ahmed II. Glaucoma and cataract surgery: two roads merging into one. Curr Opin Ophthalmol. 2016;27:51-7.

16. Michael R, Pareja-Aricò L, Rauscher FG, Barraquer RI. Cortical Cataract and Refractive Error. Ophthalmic Res. 2019;62:157-65.

17. Li L, Wan XH, Zhao GH. Meta-analysis of the risk of cataract in type 2 diabetes. BMC Ophthalmol. 2014;14:94.

18. Roodhooft JM. Leading causes of blindness worldwide. Bull Soc Belge Ophtalmol 2002:19-25.

19. Lewallen S, Williams T, Dray A, Stock B, Mathenge W, Oye J, Nkurikiye J, Kimani K, Müller A, Courtright P: Estimating incidence of vision-reducing cataract in Africa: a new model with implications for program targets. Archives of ophthalmology (Chicago, III: 1960) 2010, 128:15841589.

20. Elbieh I, Bascaran C, Blanchet K, Foster A. Trends in cataract surgical rate and resource utilisation in Egypt. Ophthalmic Epidemiol. 2018;25:351-7.

21. Lee C, Afshari N. The global state of cataract blindness. Curr Opin Ophthalmol. 2017;28:98-103.

22. Foreman J, Xie J, Keel S, van Wijngaarden P, Crowston J, Taylor HR, Dirani M. Cataract surgery coverage rates for Indigenous and non-Indigenous Australians: the National Eye Health Survey. Med J Aust. 2017;207:256-61.

23. Han X, Wu C, Yan X, Keel S, Shang X, Zhang L, He M: Are smoking intensity and cessation related to cataract surgical risk in diabetic patients? Findings from the $\mathbf{4 5}$ and Up Study. Eye 2019.

24. Nishad N, Hewage SA, Arulmoly K, Amaratunge MS, de Silva J, Kasturiratne K, Abeysundara PK, Wickramasinghe AR. Barriers for Cataract Treatment among Elderly in Sri Lanka. Curr Gerontol 
Geriatr Res. 2019;2019:6262456.

25. Odugbo OP, Mpyet CD, Chiroma MR, Aboje AO. Cataract blindness, surgical coverage, outcome, and barriers to uptake of cataract services in Plateau State, Nigeria. Middle East Afr J Ophthalmol. 2012;19:282-8.

26. Chong EW, Mehta JS. High myopia and cataract surgery. Curr Opin Ophthalmol. 2016;27:45-50.

27. Pan CW, Boey PY, Cheng CY, Saw SM, Tay WT, Wang JJ, Tan AG, Mitchell P, Wong TY. Myopia, axial length, and age-related cataract: the Singapore Malay eye study. Investig Ophthalmol Vis Sci. 2013;54:4498-502.

28. Kanthan GL, Mitchell P, Rochtchina E, Cumming RG, Wang JJ. Myopia and the long-term incidence of cataract and cataract surgery: the Blue Mountains Eye Study. Clin Exp Ophthalmol. 2014;42:347-53.

29. Pan CW, Cheng CY, Saw SM, Wang JJ, Wong TY. Myopia and age-related cataract: a systematic review and meta-analysis. Am J Ophthalmol. 2013;156:1021-33 e1021.

30. Bastawrous A, Suni AV. Thirty Year Projected Magnitude (to 2050) of Near and Distance Vision Impairment and the Economic Impact if Existing Solutions are Implemented Globally. Ophthalmic Epidemiol. 2020;27:115-20.

31. Diseases GBD, Injuries C. Global burden of 369 diseases and injuries in 204 countries and territories, 1990-2019: a systematic analysis for the Global Burden of Disease Study 2019. Lancet. 2020;396:1204-22.

32. Burga HG, Hinds CN, Lansingh VC, Samudio M, Lewallen S, Courtright P, Duerksen R, Cibils P, Zegarra M. Is the cost the primary barrier for cataract surgery in Paraguay? Arq Bras Oftalmol. 2014;77:1647.

\section{Figures}
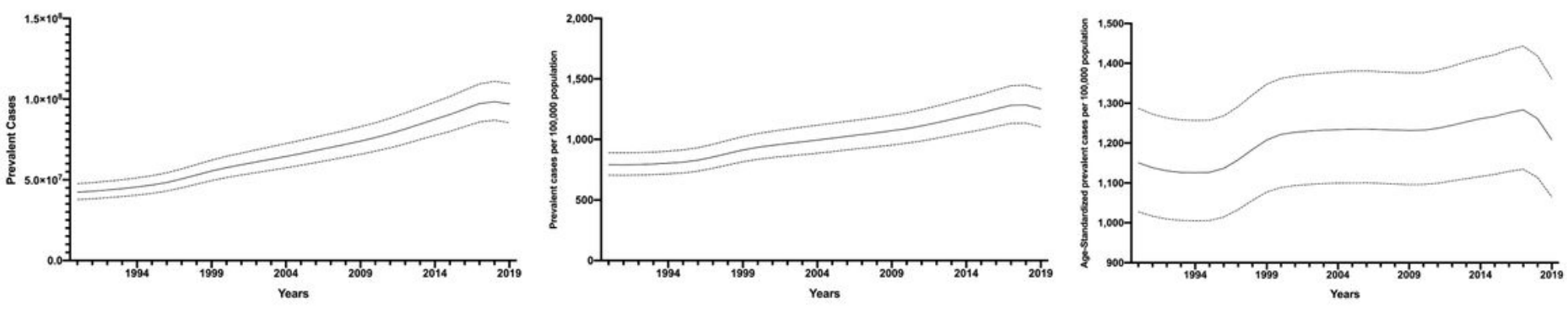

\section{Figure 1}

Prevalence of visual impairment due to cataract from 1990 to 2019 

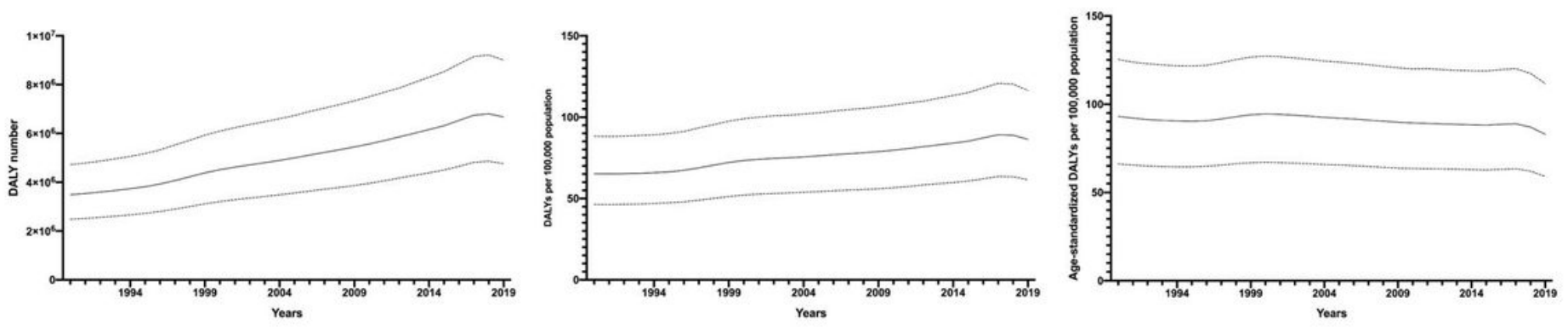

Figure 2

DALYs of visual impairment due to cataract from 1990 to 2019
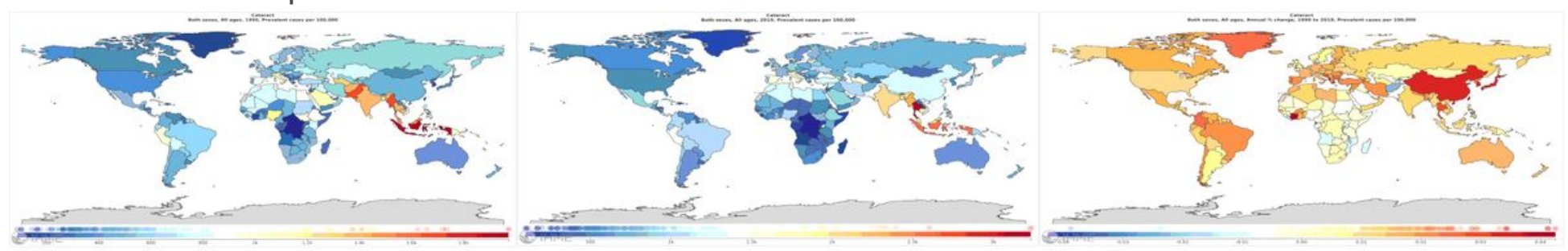

Figure 3

Distribution of prevalence of visual impairment due to cataract and the annual change in the years 1990 and 2019. 


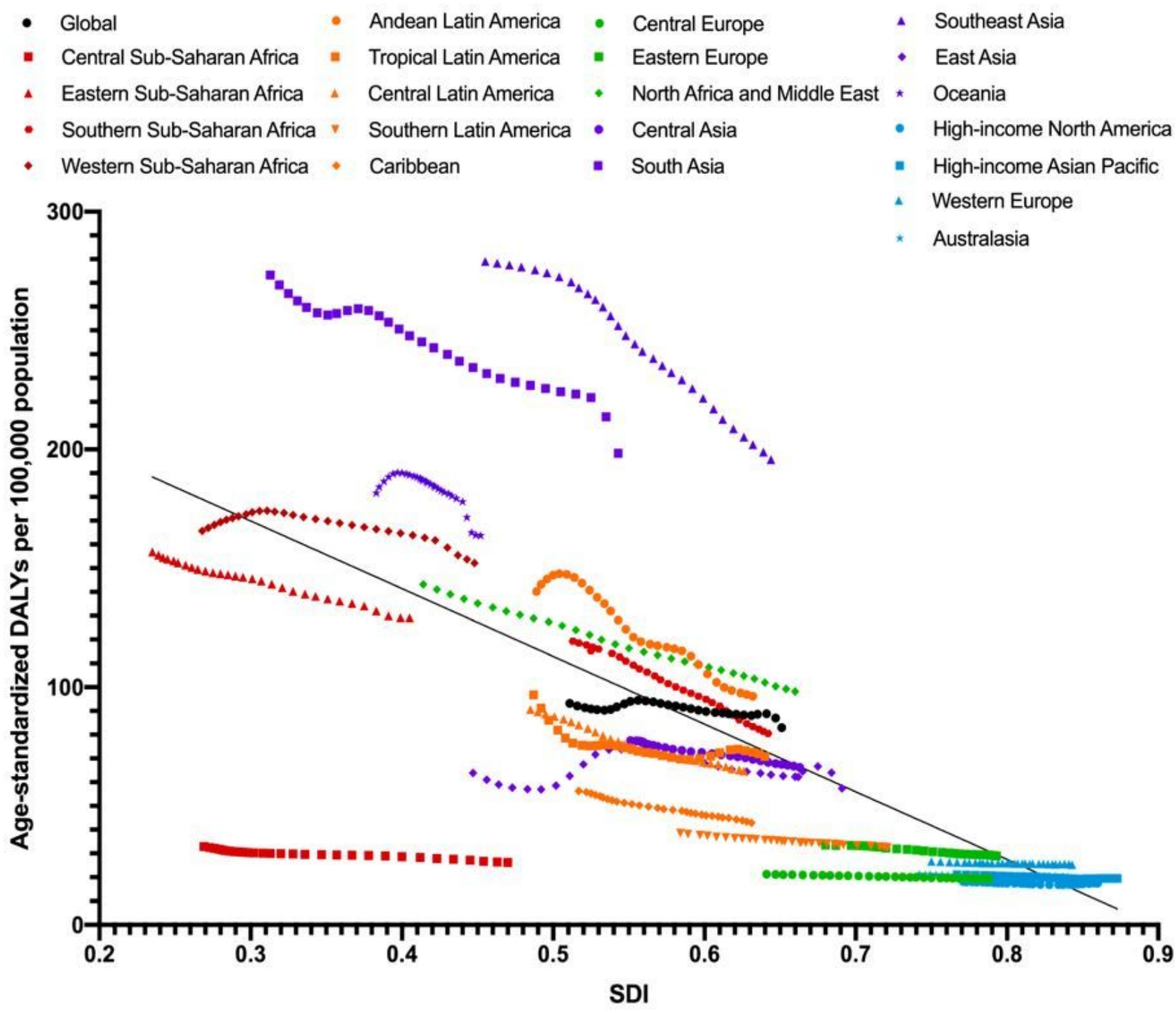

Figure 4

Age-standardized DALY rates attributable to cataract across $21 \mathrm{GBD}$ regions by socio-demographic index for both sexes combined, 1990 to 2019. 


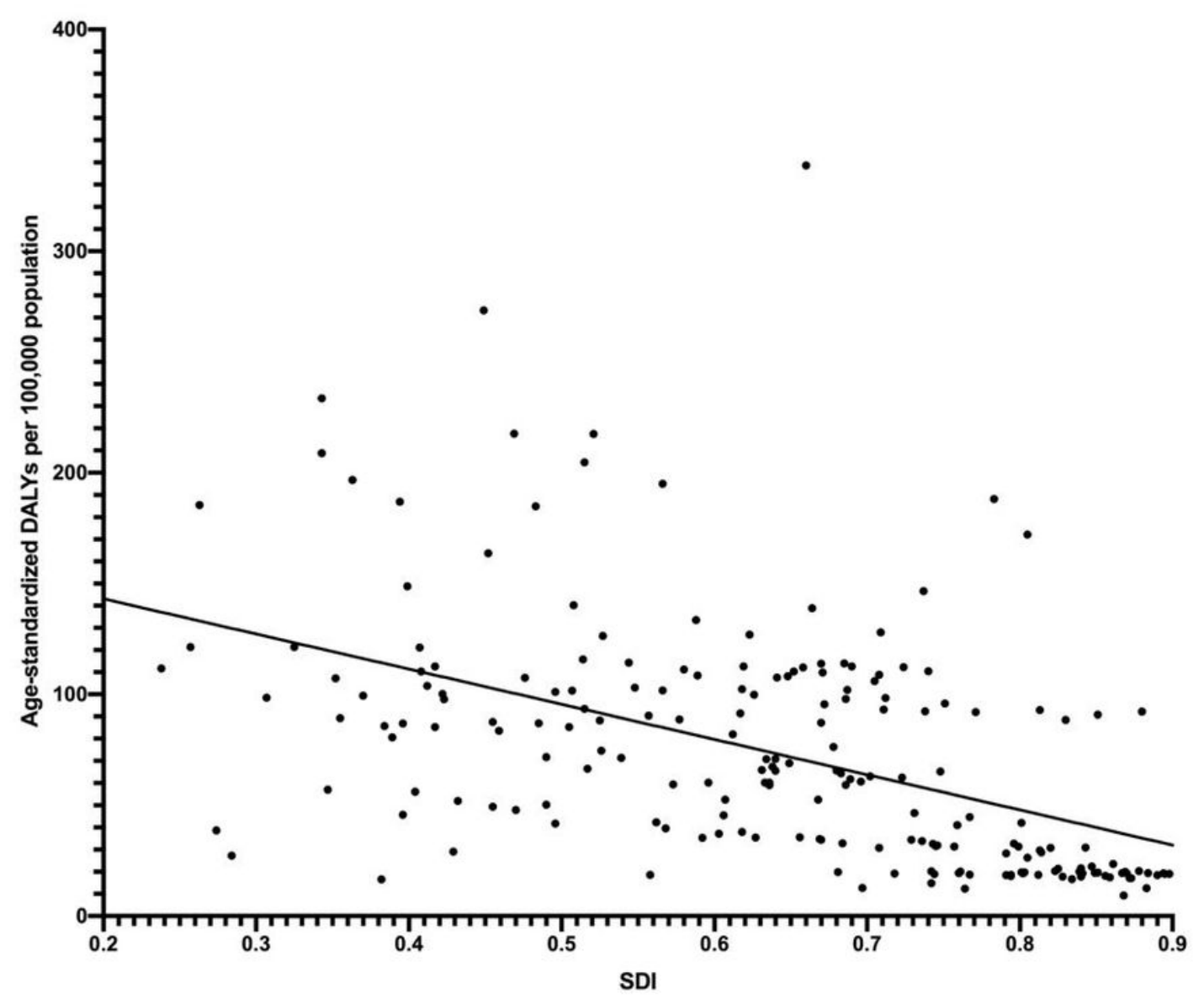

Figure 5

Age-standardized DALY rates attributable to cataract across 204 countries and territories by sociodemographic index in 2019 